\title{
INFINITE FAMILIES OF 2-DESIGNS FROM A CLASS OF LINEAR CODES RELATED TO DEMBOWSKI-OSTROM FUNCTIONS
}

\author{
RONG WANG, XIAONI DU, CUILING FAN, AND ZHIHUA NIU
}

\begin{abstract}
Due to their important applications to coding theory, cryptography, communications and statistics, combinatorial $t$-designs have been attracted lots of research interest for decades. The interplay between coding theory and $t$-designs has on going for many years. As we all known, $t$-designs can be used to derive linear codes over any finite field, as well as the supports of all codewords with a fixed weight in a code also may hold a $t$-design. In this paper, we first construct a class of linear codes from cyclic codes related to Dembowski-Ostrom functions. By using exponential sums, we then determine the weight distribution of the linear codes. Finally, we obtain infinite families of 2-designs from the supports of all codewords with a fixed weight in these codes. Furthermore, the parameters of 2-designs are calculated explicitly.
\end{abstract}

Keywords: 2-designs, linear codes, cyclic codes from Dembowski-Ostrom functions, affine-invariant codes, exponential sums, weight distributions

\section{INTRODUCTION}

We start this article by introducing the relevant conception to $t$-designs. Let $v, k, \lambda$, and $t$ be positive integers such that $v>k \geq t$. Let $P$ be a set with cardinality $|\mathcal{P}|=v$ and $\mathcal{B}$ be a multi-set of $k$-subsets of $\mathcal{P}$ with $|\mathcal{B}|=b$. The elements of $\mathcal{P}$ are called as points and that of $\mathcal{B}$ as blocks. A $t-(v, k, \lambda)$ design is a pair $\mathbb{D}=(\mathcal{P}, \mathcal{B})$ such that each block contains exactly $k$ points, and every set of $t$ distinct points is contained in exactly $\lambda$ blocks. A $t-(v, k, \lambda)$ design without repeated blocks is called a simple $t$-design. A $t$-design is called symmetric if $v=b$ and trivial if $k=t$ or $k=v$. In the following, we pay only attention to simple $t$-designs with $t<k<v$. When $t \geq 2$, we call the $t-(v, k, 1)$ design a Steiner system. Furthermore, the number of blocks in a $t$-design is

$$
b=\frac{\lambda\left(\begin{array}{l}
v \\
t
\end{array}\right)}{\left(\begin{array}{l}
k \\
t
\end{array}\right)} .
$$

Date: December 17, 2019. 
The study of $t$-designs as a mathematical discipline has been a very interesting subject for many years due to their wide applications in coding theory and analysis of statistical experiments. $t$-Designs have many other applications as well, such as cryptography, communications and other engineering areas. It is well known that the interplay between linear codes and $t$-designs has been attracted a lot of attention for decades. A linear code over any finite field can be derived from the incidence matrix of a $t$-design and much progress has been made (see for example [1, 11, 25, 26]). On the other hand, both linear and nonlinear codes can hold $t$-designs and some of 2-designs and 3-designs are derived from codes, we refer the readers to [1, 4, 5, 6, 19, 20, 23, 24, 25, 26, 28] for examples. The main technical idea is based on the support design, that is, if one index the coordinates of a codeword in a code $C$ by $(0,1, \ldots, n-1)$ and let $\mathcal{P}=(0,1, \ldots, n-1)$. And so the pair $\left(\mathcal{P}, \mathcal{B}_{i}\right)$ may be a $t-(n, i, \lambda)$ design for some positive $\lambda$, where $\mathcal{B}_{i}$ is the set of the supports of all codewords with weight $i$ in $C$ for each $i$ with $A_{i} \neq 0$ [9] (we will describe the definition below).

There exist two classical approaches to obtain infinitely families of $t$ designs from linear codes. The first is to employ the Assmus-Mattson Theorem given in [2, 3] and we refer the readers to [8, 9] for details. The second is to study the automorphism group of a linear code $C$. That is, if the permutation part of the automorphism group acts $t$-transitively on a code $\mathcal{C}$, then the code $C$ holds $t$-designs [1, 23]. Only a small amount of work in the direction has been done. For example, Ding et al. [10] and Du et al. [12, 13, 14] have derived infinite families of 3-designs and 2-designs from several different classes of affine-invariant codes, respectively. In this paper, we will obtain infinite families of 2-designs by studying the automorphism group of a class of linear codes we construct.

The remainder of this paper is organized as follows. In Section 2, we promote the definition of the linear codes we discuss, which are derived from cyclic codes related to a class of Dembowski-Ostrom functions. In Section 3, we introduce some notation and preliminary results on affine invariant codes, 2-designs, exponential sums and cyclotomic fields, which will be used in subsequent sections. In Section 4, we determine explicitly the weight distribution of the codes we defined by using certain exponential sums and obtain infinite families of 2-designs and their parameters. Section 5 proves the main results. Furthermore, we also use Magma programs to give some examples. And Section 6 concludes the paper.

\section{LINEAR CODES AND OUR CONSTRUCTION}

Let $p$ be an odd prime and $m$ any positive integer. Let $\mathbb{F}_{q}$ denote the finite field with $q=p^{m}$ elements and $\mathbb{F}_{q}^{*}=\mathbb{F}_{q} \backslash\{0\}$. An $[n, k, \delta]$ linear code 
$\mathcal{C}$ over $\mathbb{F}_{p}$ is called cyclic code if each codeword $\left(c_{0}, c_{1}, \ldots, c_{n-1}\right) \in \mathcal{C}$ implies $\left(c_{n-1}, c_{0}, c_{1}, \ldots, c_{n-2}\right) \in \mathcal{C}$. Any cyclic code $\mathcal{C}$ can be expressed as $\mathcal{C}=\langle g(x)\rangle$, where $g(x)$ is monic and has the least degree. The polynomial $g(x)$ is called the generator polynomial and $h(x)=\left(x^{n}-1\right) / g(x)$ is referred to as the parity-check polynomial of $C$. The dual of a cyclic code $\mathcal{C}$, denoted by $\mathcal{C}^{\perp}$, is a cyclic code with length $n$, dimension $n-k$ and generator polynomial $x^{k} h\left(x^{-1}\right) / h(0)$. The extended code $\bar{C}$ of $\mathcal{C}$ is defined by

$$
\overline{\mathcal{C}}=\left\{\left(c_{0}, c_{1}, \ldots, c_{n}\right) \in \mathbb{F}_{p}^{n+1}:\left(c_{0}, c_{1}, \ldots, c_{n-1}\right) \in \mathcal{C} \text { with } \sum_{i=0}^{n} c_{i}=0\right\}
$$

Let $A_{i}$ be the number of codewords with Hamming weight $i$ in a code $C$. The weight enumerator of $\mathcal{C}$ is defined by $\sum_{i=0}^{n} A_{i} z^{i}$, and the sequence $\left(1, A_{1}, \ldots, A_{n}\right)$ is called the weight distribution of the code $C$. The weight distribution is an important research object in coding theory because it contains some crucial information of the code, for example, the minimum weight, which determines the error correction ability of the code. If the number of nonzero $A_{i}$ 's with $1 \leq i \leq n$ is $w$, then we call $C$ a $w$-weight code.

If $\mathbf{c}=\left(c_{0}, c_{1}, \ldots, c_{n-1}\right)$ is a codeword in a code $\mathcal{C}$, then support of $\mathbf{c}$ is defined by

$$
\operatorname{Suppt}(\mathbf{c})=\left\{0 \leq i \leq n-1: c_{i} \neq 0\right\} \subseteq\{0,1, \ldots, n-1\} .
$$

For any integer $0 \leq j<n$, the $p$-cyclotomic coset of $j$ modulo $n$ is defined by

$$
C_{j}=\left\{j p^{i} \quad(\bmod n): 0 \leq i \leq \ell_{j}-1\right\},
$$

where $\ell_{j}$ is the smallest positive integer such that $j \equiv j p^{\ell_{j}}(\bmod n)$. The smallest integer in $C_{j}$ is called the coset leader of $C_{j}$. For a cyclic code $C$, its generator polynomial $g(x)$ can be written as $g(x)=\prod_{j} \prod_{e \in C_{j}}(x-$ $\left.\alpha^{e}\right)$, where $j$ runs through some coset leaders of the $p$-cyclotomic cosets $C_{j}$ modulo $n$. The set $T=\bigcup_{j} C_{j}$ is referred to as the defining set of $C$, which is the union of these $p$-cyclotomic cosets.

Below, let $\alpha$ be a primitive element of $\mathbb{F}_{q}, h_{i}(x)$ be the minimal polynomials of $\alpha^{i}$ over $\mathbb{F}_{p}$ and $\operatorname{Tr}$ denote the trace function from $\mathbb{F}_{q}$ onto $\mathbb{F}_{p}$ given by

$$
\operatorname{Tr}(x)=x+x^{p}+\cdots+x^{p^{m-1}}, \text { for any } x \in \mathbb{F}_{q} .
$$

Denote $\mathbb{C}$ by the cyclic code with length $n=q-1$ and parity-check polynomial $h_{1}(x) h_{p^{l}+1}(x) h_{p^{3 l+1}}(x)$ related to a class of Dembowski-Ostrom functions [21], where $1 \leq l \leq m-1$ and $l \notin\left\{\frac{m}{6}, \frac{m}{4}, \frac{m}{2}, \frac{3 m}{4}, \frac{5 m}{6}\right\}$. We introduce the 
linear code $\overline{\mathbb{C}}^{\perp}$ by

$$
\begin{aligned}
{\overline{\mathbb{C}^{\perp}}}^{\perp}:=\left\{\left(\operatorname { T r } \left(a x^{p^{3 l}+1}+b x^{p^{l}+1}+\right.\right.\right. & \left.c x)_{x \in \mathbb{F}_{q}}+h\right): \\
& \left.a, b, c \in \mathbb{F}_{q}, h \in \mathbb{F}_{p}\right\} .
\end{aligned}
$$

In [21], Luo et al. examined the weight distribution of cyclic code $\mathbb{C}$. In this paper, we will determine the weight distribution of the linear code $\overline{\mathbb{C}}^{\perp}$ and then obtain infinitely families 2-designs from the codewords with nonzero weight in $\overline{\mathbb{C}}^{\perp}$. If $m=3 d, \overline{\mathbb{C}}^{\perp}$ is the code we discussed in [12], thus we suppose $m \neq 3 d$ in this paper.

\section{PRELIMINARIES}

In this section, we summarize some standard notation and basic facts on affine-invariant codes, 2-designs, exponential sums and cyclotomic fields.

3.1. Some notation. For convenience, we adopt the following notation unless otherwise stated in this paper.

- $m \geq 3$ and $l$ are positive integers with $1 \leq l \leq m-1$ and $l \notin\left\{\frac{m}{6}, \frac{m}{4}, \frac{m}{2}, \frac{3 m}{4}\right.$, $\left.\frac{5 m}{6}\right\}$.

- $d^{\prime}=\operatorname{gcd}(m, 2 l)$ and $d=\operatorname{gcd}(m, l)$.

- If $m$ is even, $s=m / 2$ and $\mu=(-1)^{s / d}$.

- $p$ is an odd prime and $p^{*}=(-1)^{\frac{p-1}{2}} p$.

- $q=p^{m}, n=p^{m}-1$ and $\varepsilon=(-1)^{v}$, where $v=0,1$.

- $\zeta_{N}=e^{2 \pi \sqrt{-1} / N}$ is a primitive $N$-th root of unity for any integer $N \geq$ 2.

- $\eta$ and $\eta^{\prime}$ are the quadratic characters of $\mathbb{F}_{q}^{*}$ and $\mathbb{F}_{p}^{*}$, respectively (we will give the definition below).

3.2. Affine-invariant codes and 2-designs. We begin this subsection by introducing the affine-invariant code. The set of coordinate permutations that map a code $C$ to itself forms a group, which we call the permutation automorphism group of $C$ and denote by PAut $(C)$. We define the affine group $G A_{1}\left(\mathbb{F}_{q}\right)$ by the set of all permutations

$$
\sigma_{a, b}: x \mapsto a x+b
$$

of $\mathbb{F}_{q}$, where $a \in \mathbb{F}_{q}^{*}$ and $b \in \mathbb{F}_{q}$. An affine-invariant code is an extended cyclic code $\bar{C}$ over $\mathbb{F}_{p}$ such that $G A_{1}\left(\mathbb{F}_{q}\right) \subseteq P A u t(\bar{C})[16]$.

The $p$-adic expansion of each $s \in \mathcal{P}$ is given by

$$
s=\sum_{i=0}^{m-1} s_{i} p^{i}, 0 \leq s_{i} \leq p-1,0 \leq i \leq m-1 .
$$


For any $r=\sum_{i=0}^{m-1} r_{i} p^{i} \in \mathcal{P}$, we say that $r \preceq s$ if $r_{i} \leq s_{i}$ for all $0 \leq i \leq m-1$. Clearly, $r \preceq s$ implies that $r \leq s$.

For some applications, an important question is to decide whether a given linear codes $\bar{C}$ and $\bar{C}^{\perp}$ are affine-invariant or not. The answers are provided by the following lemma. More precisely, the first item given by Kasami et al. [18] points out that one can determine whether a given extended primitive cyclic code $\bar{C}$ is affine-invariant by analysing the properties of its defining set and the second item proposed by Ding [7] shows that one can get new affine-invariant code from the known ones.

Lemma 1. Let $\bar{C}$ be an extended cyclic code of length $p^{m}$ over $\mathbb{F}_{p}$ with defining set $\bar{T}$.

(1) [18] $\bar{C}$ is affine-invariant if and only if whenever $s \in \bar{T}$ then $r \in \bar{T}$ for all $r \in \mathcal{P}$ with $r \preceq s$.

(2) [7] The dual of an affine-invariant code $\bar{C}$ over $\mathbb{F}_{p}$ of length $n+1$ is also affine-invariant.

Theorem 2. [7] For each $i$ with $A_{i} \neq 0$ in an affine-invariant code $\overline{\mathcal{C}}$, the supports of the codewords of weight i form a 2-design.

Theorem 2 is very attractive in the sense that they determine the existence of 2-designs. The following theorem, describe the relation of all codewords with the same support in a linear code $C$, will be used together with Eq.(1) to calculate the parameters of 2-designs later.

Theorem 3. [7] Let $\mathcal{C}$ be a linear code over $\mathbb{F}_{p}$ with length $n$ and minimum weight $\delta$. Let $w$ be the largest integer with $w \leq n$ satisfying

$$
w-\left\lfloor\frac{w+p-2}{p-1}\right\rfloor<\delta .
$$

Let $\mathbf{c}_{\mathbf{1}}$ and $\mathbf{c}_{\mathbf{2}}$ be two codewords of weight $i$ with $\delta \leq i \leq w$ and Suppt $\left(\mathbf{c}_{\mathbf{1}}\right)=$ Suppt $\left(\mathbf{c}_{2}\right)$. Then $\mathbf{c}_{1}=$ a $\mathbf{c}_{2}$ for some $a \in \mathbb{F}_{p}^{*}$.

3.3. Exponential sums. Generally, the weight of each codeword in a cyclic code $C$ can be expressed by certain exponential sums so that the weight distribution of $C$ can be determined when the exponential sums could be computed explicitly (see [15, 27, 29, 30] and the references therein).

An additive character of $\mathbb{F}_{q}$ is a nonzero function $\chi$ from $\mathbb{F}_{q}$ to the set of complex numbers of absolute value 1 such that $\chi(x+y)=\chi(x) \chi(y)$ for any pair $(x, y) \in \mathbb{F}_{q}^{2}$. For each $u \in \mathbb{F}_{q}$, the function

$$
\chi_{u}(v)=\zeta_{p}^{\operatorname{Tr}(u v)}, v \in \mathbb{F}_{q},
$$

denotes an additive character of $\mathbb{F}_{q}$. We call $\chi_{1}$ the canonical additive character of $\mathbb{F}_{q}$ and we have $\chi_{u}(x)=\chi_{1}(u x)$ for all $u \in \mathbb{F}_{q}[22]$. 
For each $j=0,1, \ldots, q-2$, a multiplicative character of $\mathbb{F}_{q}$ is defined by the function $\psi_{j}\left(\alpha^{k}\right)=\zeta_{q-1}^{j k}$, for $k=0,1, \ldots, q-2$. We denote by $\eta:=\psi_{\frac{q-1}{2}}$ which is called the quadratic character of $\mathbb{F}_{q}$. Similarly, we may define the quadratic character $\eta^{\prime}$ of $\mathbb{F}_{p}$. We extend these quadratic characters by letting $\eta(0)=0$ and $\eta^{\prime}(0)=0$.

The Gauss sum $G\left(\eta^{\prime}, \chi_{1}^{\prime}\right)$ over $\mathbb{F}_{p}$ is defined by $G\left(\eta^{\prime}, \chi_{1}^{\prime}\right)=\sum_{v \in \mathbb{F}_{p}^{*}} \eta^{\prime}(v) \chi_{1}^{\prime}(v)=$ $\sum_{v \in \mathbb{F}_{p}} \eta^{\prime}(v) \chi_{1}^{\prime}(v)$, where $\chi_{1}^{\prime}$ is the canonical additive character of $\mathbb{F}_{p}$. The following Lemma 4 related to Gauss sum is essential to determine the value of Eq.(3).

Lemma 4. [22] With the notation above, we have

$$
G\left(\eta^{\prime}, \chi_{1}^{\prime}\right)=\sqrt{(-1)}^{\left(\frac{p-1}{2}\right)^{2}} \sqrt{p}=\sqrt{p^{*}}
$$

To determine the weight distribution of the code $\overline{\mathbb{C}}^{\perp}$, we introduce the following function

$$
S(a, b, c)=\sum_{x \in \mathbb{F}_{q}} \zeta_{p}^{T r\left(a x^{p^{3 l}+1}+b x^{p^{l}+1}+c x\right)}, \quad a, b, c \in \mathbb{F}_{q} .
$$

For the value distribution of $S(a, b, c)$, we have the following results given by Luo et al..

Lemma 5. [21] For $m \geq 3$ and $j \in \mathbb{F}_{p}^{*}$, the value distribution of $\{S(a, b, c)$ : $\left.a, b, c \in \mathbb{F}_{q}\right\}$ defined in Eq.(3) is given in Table 8 if $d^{\prime}=d$ is odd, in Table 9 if $d^{\prime}=d$ is even and in Table 10 if $d^{\prime}=2 d$, respectively (see Tables 8,9 and 10 in Appendix I).

It is clear that $d^{\prime}=d$ is odd implies $m$ is odd.

3.4. Cyclotomic fields. We state the following basic facts on Galois group of cyclotomic fields $\mathbb{Q}\left(\zeta_{p}\right)$ since $S(a, b, c)$ is element in $\mathbb{Q}\left(\zeta_{p}\right)$.

Lemma 6. [17] Let $\mathbb{Z}$ be the rational integer ring and $\mathbb{Q}$ be the rational field.

(1) The ring of integers in $K=\mathbb{Q}\left(\zeta_{p}\right)$ is $O_{k}=\mathbb{Z}\left[\zeta_{p}\right]$ and $\left\{\zeta_{p}^{i}: 1 \leq i \leq\right.$ $p-1\}$ is an integral basis of $O_{k}$.

(2) The filed extension $K / \mathbb{Q}$ is Galois of degree $p-1$ and the Galois $\operatorname{group} \operatorname{Gal}(K / \mathbb{Q})=\left\{\sigma_{y}: y \in(\mathbb{Z} / p \mathbb{Z})^{*}\right\}$, where the automorphism $\sigma_{y}$ of $K$ is defined by $\sigma_{y}\left(\zeta_{p}\right)=\zeta_{p}^{y}$.

(3) $K$ has a unique quadratic subfield $L=\mathbb{Q}\left(\sqrt{p^{*}}\right)$. For $1 \leq y \leq p-$ $1, \sigma_{y}\left(\sqrt{p^{*}}\right)=\eta^{\prime}(y) \sqrt{p^{*}}$. Therefore, the Galois group $\operatorname{Gal}(L / \mathbb{Q})$ is $\left\{1, \sigma_{\gamma}\right\}$, where $\gamma$ is any quadratic nonresidue in $\mathbb{F}_{p}$. 
TABLE 1. The weight distribution of ${\overline{\mathbb{C}^{\perp}}}^{\perp}$ when $d^{\prime}=d$ is odd

\begin{tabular}{ll}
\hline Weight & Multiplicity \\
\hline 0 & 1 \\
$p^{m-1}(p-1)$ & $p\left(p^{2 m+2 d-1}+p^{2 m+d}-p^{2 m+d-1}\right.$ \\
& $-p^{2 m}-p^{2 m-1}+p^{2 m-2 d}-p^{2 m-3 d}$ \\
& $+p^{2 m-3 d-1}+p^{m+2 d-1}-p^{m}+$ \\
& $\left.p^{m-2 d}-p^{m-2 d-1}+p^{2 d}-1\right)$ \\
& $\left(p^{m}-1\right) /\left(p^{2 d}-1\right)$ \\
$p^{\frac{m-1}{2}}\left(p^{\frac{m+1}{2}}-p^{\frac{m-1}{2}}+\varepsilon(-1)^{\frac{p-1}{2}}\right)$ & $\frac{p^{m+2 d}(p-1)\left(p^{m}-p^{m-d}-p^{m-2 d}+1\right)\left(p^{m}-1\right)}{2\left(p^{2 d}-1\right)}$ \\
$p^{\frac{m+d-2}{2}}(p-1)\left(p^{\frac{m-d}{2}}+\varepsilon\right)$ & $\frac{1}{2} p^{\frac{3 m-3 d}{2}}\left(p^{\frac{m-d}{2}}-\varepsilon\right)\left(p^{m}-1\right)$ \\
$p^{\frac{m+d-2}{2}}\left(p^{\frac{m-d+2}{2}}-p^{\frac{m-d}{2}}+\varepsilon\right)$ & $\frac{1}{2} p^{\frac{3 m-3 d}{2}}(p-1)\left(p^{\frac{m-d}{2}}+\varepsilon\right)\left(p^{m}-1\right)$ \\
$p^{\frac{m+2 d-1}{2}}\left(p^{\frac{m-2 d+1}{2}}-p^{\frac{m-2 d-1}{2}}+\varepsilon(-1)^{\frac{p-1}{2}}\right)$ & $\frac{p^{m-2 d}(p-1)\left(p^{m-d}-1\right)\left(p^{m}-1\right)}{2\left(p^{2 d}-1\right)}$ \\
$p^{m}$ & $p-1$ \\
\hline
\end{tabular}

\section{MAIN RESUlTS}

In the section, we only present the main results on the weight distribution of the code $\overline{\mathbb{C}}^{\perp}$ and the corresponding 2-designs. The proofs will be presented in subsequent section.

Theorem 7. For $m \geq 6$, the weight distribution of the code ${\overline{\mathbb{C}^{\perp}}}^{\perp}$ over $\mathbb{F}_{p}$ with length $n+1$ and dimension $\operatorname{dim}\left(\overline{\mathbb{C}}^{\perp}\right)=3 m+1$ is given in Table 1 when $d^{\prime}=d$ is odd, in Table 2 when $d^{\prime}=d$ is even and in Table 3 when $d^{\prime}=2 d$, respectively.

One can see that the code is at most 10-weight if $d^{\prime}=d$ is odd or $d^{\prime}=2 d$, at most 14-weight if $d^{\prime}=d$ is even.

Theorem 8. Let $m \geq 6$ be a positive integer. Then the supports of the all codewords of weight $i>0$ in ${\overline{\mathbb{C}^{\perp}}}^{\perp}$ form a 2-design, provided that $A_{i} \neq 0$.

For the parameters of the 2-designs derived from ${\overline{\mathbb{C}^{\perp}}}^{\perp}$, we have the following.

Theorem 9. Let $\mathcal{B}$ be the set of the supports of the codewords of $\overline{\mathbb{C}}^{\perp}$ with weight $i$, where $A_{i} \neq 0$. Then for $m \geq 6, \overline{\mathbb{C}}^{\perp}$ holds $2-\left(p^{m}, i, \lambda\right)$ designs for the following pairs:

(1) If $d^{\prime}=d$ is odd, then 
TABLE 2. The weight distribution of $\overline{\mathbb{C}}^{\perp}$ when $d^{\prime}=d$ is even

\begin{tabular}{ll}
\hline Weight & Multiplicity \\
\hline 0 & 1 \\
$p^{s-1}(p-1)\left(p^{s}+\varepsilon\right)$ & $\frac{p^{m+2 d}\left(p^{m}-p^{m-d}-p^{m-2 d}+1\right)\left(p^{m}-1\right)}{2\left(p^{2 d}-1\right)}$ \\
$p^{s-1}\left(p^{s+1}-p^{s}+\varepsilon\right)$ & $\frac{p^{m+2 d}(p-1)\left(p^{m}-p^{m-d}-p^{m-2 d}+1\right)\left(p^{m}-1\right)}{2\left(p^{2 d}-1\right)}$ \\
$p^{\frac{m+d-2}{2}}(p-1)\left(p^{\frac{m-d}{2}}+\varepsilon\right)$ & $\frac{1}{2} p^{\frac{3 m-3 d}{2}}\left(p^{\frac{m-d}{2}}-\varepsilon\right)\left(p^{m}-1\right)$ \\
$p^{\frac{m+d-2}{2}}\left(p^{\frac{m-d+2}{2}}-p^{\frac{m-d}{2}}+\varepsilon\right)$ & $\frac{1}{2} p^{\frac{3 m-3 d}{2}}(p-1)\left(p^{\frac{m-d}{2}}+\varepsilon\right)\left(p^{m}-1\right)$ \\
$p^{\frac{m+2 d-2}{2}}(p-1)\left(p^{\frac{m-2 d}{2}}+\varepsilon\right)$ & $\frac{1}{2} p^{m-2 d}\left(p^{m-d}-1\right)\left(p^{m}-1\right) /\left(p^{2 d}-1\right)$ \\
$p^{\frac{m+2 d-2}{2}}\left(p^{\frac{m-2 d+2}{2}}-p^{\frac{m-2 d}{2}}+\varepsilon\right)$ & $\frac{p^{m-2 d}(p-1)\left(p^{m-d}-1\right)\left(p^{m}-1\right)}{2\left(p^{2 d}-1\right)}$ \\
$p^{m-1}(p-1)$ & $p\left(p^{2 m-d}-p^{2 m-2 d}+p^{2 m-3 d}-p^{m-2 d}\right.$ \\
$p^{m}$ & $+1)\left(p^{m}-1\right)$ \\
\hline
\end{tabular}

- $(i, \lambda)=\left(p^{m-1}(p-1),\left(p^{m}-p^{m-1}-1\right)\left(p^{2 m+2 d-1}+p^{2 m+d}-p^{2 m+d-1}\right.\right.$ $-p^{2 m}-p^{2 m-1}+p^{2 m-2 d}-p^{2 m-3 d}+p^{2 m-3 d-1}+p^{m+2 d-1}-p^{m}+$ $\left.\left.p^{m-2 d}-p^{m-2 d-1}+p^{2 d}-1\right) /\left(p^{2 d}-1\right)\right)$;

- $(i, \lambda)=\left(p^{\frac{m-1}{2}}\left(p^{\frac{m+1}{2}}-p^{\frac{m-1}{2}}+\varepsilon(-1)^{\frac{p-1}{2}}\right), p^{\frac{m+4 d-1}{2}}\left(p^{\frac{m+1}{2}}-p^{\frac{m-1}{2}}+\varepsilon\right.\right.$ $\left.(-1)^{\frac{p-1}{2}}\right)\left[p^{\frac{m-1}{2}}\left(p^{\frac{m+1}{2}}-p^{\frac{m-1}{2}}+\varepsilon(-1)^{\frac{p-1}{2}}\right)-1\right]\left(p^{m}-p^{m-d}-p^{m-2 d}+\right.$ 1) $\left./ 2\left(p^{2 d}-1\right)\right)$;

- $(i, \lambda)=\left(p^{\frac{m+d-2}{2}}(p-1)\left(p^{\frac{m-d}{2}}+\varepsilon\right), \frac{1}{2} p^{m-d-1}\left[p^{\frac{m+d-2}{2}}(p-1)\left(p^{\frac{m-d}{2}}+\right.\right.\right.$ $\left.\varepsilon)-1]\left(p^{m-d}-1\right)\right)$;

- $(i, \lambda)=\left(p^{\frac{m+d-2}{2}}\left(p^{\frac{m-d+2}{2}}-p^{\frac{m-d}{2}}+\varepsilon\right), \frac{1}{2} p^{m-d-1}\left(p^{\frac{m-d+2}{2}}-p^{\frac{m-d}{2}}+\varepsilon\right)[\right.$ $\left.\left.p^{\frac{m+d-2}{2}}\left(p^{\frac{m-d+2}{2}}-p^{\frac{m-d}{2}}+\varepsilon\right)-1\right]\left(p^{\frac{m-d}{2}}+\varepsilon\right)\right)$;

- $(i, \lambda)=\left(p^{\frac{m+2 d-1}{2}}\left(p^{\frac{m-2 d+1}{2}}-p^{\frac{m-2 d-1}{2}}+\varepsilon(-1)^{\frac{p-1}{2}}\right), \frac{1}{2} p^{\frac{m-2 d-1}{2}}\left(p^{\frac{m-2 d+1}{2}}-\right.\right.$ $\left.p^{\frac{m-2 d-1}{2}}+\varepsilon(-1)^{\frac{p-1}{2}}\right)\left[p^{\frac{m+2 d-1}{2}}\left(p^{\frac{m-2 d+1}{2}}-p^{\frac{m-2 d-1}{2}}+\varepsilon(-1)^{\frac{p-1}{2}}\right)-1\right]$ $\left.\left(p^{m-d}-1\right) /\left(p^{2 d}-1\right)\right)$;

(2) If $d^{\prime}=d$ is even and $m \geq 8$, then

- $(i, \lambda)=\left(p^{s-1}(p-1)\left(p^{s}+\varepsilon\right), p^{s+2 d-1}\left(p^{s}+\varepsilon\right)\left[p^{s-1}(p-1)\left(p^{s}+1\right)-\right.\right.$ $\left.1]\left(p^{m}-p^{m-d}-p^{m-2 d}+1\right) / 2\left(p^{2 d}-1\right)\right)$;

- $(i, \lambda)=\left(p^{s-1}\left(p^{s+1}-p^{s}+\varepsilon\right), \frac{1}{2} p^{s+2 d-1}\left(p^{s+1}-p^{s}+\varepsilon\right)\left[p^{s-1}\left(p^{s+1}-\right.\right.\right.$ $\left.\left.\left.p^{s}+\varepsilon\right)-1\right]\left(p^{m}-p^{m-d}-p^{m-2 d}+1\right) /\left(p^{2 d}-1\right)\right)$;

- $(i, \lambda)=\left(p^{\frac{m+d-2}{2}}(p-1)\left(p^{\frac{m-d}{2}}+\varepsilon\right), \frac{1}{2} p^{m-d-1}\left(p^{m-d}-1\right)\left[p^{\frac{m+d-2}{2}}(p-\right.\right.$ 1) $\left.\left.\left(p^{\frac{m-d}{2}}+\varepsilon\right)-1\right]\right)$; 
TABLE 3. The weight distribution of $\overline{\mathbb{C}}^{\perp}$ when $d^{\prime}=2 d$

\begin{tabular}{|c|c|}
\hline Weight & Multiplicity \\
\hline 0 & 1 \\
\hline$p^{s-1}(p-1)\left(p^{s}-\mu\right)$ & $\begin{array}{l}p^{m}\left(p^{m+6 d}-p^{m+4 d}-p^{m+d}+\mu p^{s+5 d}-\right. \\
\left.\mu p^{s+4 d}+p^{6 d}\right)\left(p^{m}-1\right) /\left(p^{d}+1\right)\left(p^{2 d}-1\right) \\
\left(p^{3 d}+1\right)\end{array}$ \\
\hline$p^{s-1}\left(p^{s+1}-p^{s}+\mu\right)$ & $\begin{array}{l}p^{m}(p-1)\left(p^{m+6 d}-p^{m+4 d}-p^{m+d}+\mu p^{s+5 d}-\right. \\
\left.\mu p^{s+4 d}+p^{6 d}\right)\left(p^{m}-1\right) /\left(p^{d}+1\right)\left(p^{2 d}-1\right) \\
\left(p^{3 d}+1\right)\end{array}$ \\
\hline$p^{s+d-1}(p-1)\left(p^{s-d}+\mu\right)$ & $\begin{array}{l}p^{m-2 d}\left(p^{m+3 d}+p^{m+2 d}-p^{m}-p^{m-d}-p^{m-2 d}-\right. \\
\left.\mu p^{s+3 d}+\mu p^{s}+p^{3 d}\right)\left(p^{m}-1\right) /\left(p^{d}+1\right)^{2}\left(p^{2 d}-1\right)\end{array}$ \\
\hline$p^{s+d-1}\left(p^{s-d+1}-p^{s-d}-\mu\right)$ & $\begin{array}{l}p^{m-2 d}(p-1)\left(p^{m+3 d}+p^{m+2 d}-p^{m}-p^{m-d}-\right. \\
\left.p^{m-2 d}-\mu p^{s+3 d}+\mu p^{s}+p^{3 d}\right)\left(p^{m}-1\right) /\left(p^{d}+1\right)^{2} \\
\left(p^{2 d}-1\right)\end{array}$ \\
\hline$p^{s+2 d-1}(p-1)\left(p^{s-2 d}-\mu\right)$ & $\begin{array}{l}p^{m-4 d}\left(p^{s-d}+\mu\right)\left(p^{s+d}+p^{s}-p^{s-2 d}-\mu p^{d}\right) \\
\left(p^{m}-1\right) /\left(p^{d}+1\right)^{2}\left(p^{2 d}-1\right)\end{array}$ \\
\hline$p^{s+2 d-1}\left(p^{s-2 d+1}-p^{s-2 d}+\mu\right)$ & $\begin{array}{l}p^{m-4 d}(p-1)\left(p^{s-d}+\mu\right)\left(p^{s+d}+p^{s}-p^{s-2 d}-\right. \\
\left.\mu p^{d}\right)\left(p^{m}-1\right) /\left(p^{d}+1\right)^{2}\left(p^{2 d}-1\right)\end{array}$ \\
\hline$p^{s+3 d-1}(p-1)\left(p^{s-3 d}+\mu\right)$ & $\begin{array}{l}p^{m-6 d}\left(p^{s-2 d}-\mu\right)\left(p^{s-d}+\mu\right)\left(p^{m}-1\right) /\left(p^{d}+1\right) \\
\left(p^{2 d}-1\right)\left(p^{3 d}+1\right)\end{array}$ \\
\hline$p^{s+3 d-1}\left(p^{s-3 d+1}-p^{s-3 d}-\mu\right)$ & $\begin{array}{l}p^{m-6 d}(p-1)\left(p^{s-2 d}-\mu\right)\left(p^{s-d}+\mu\right)\left(p^{m}-1\right) / \\
\left(p^{d}+1\right)\left(p^{2 d}-1\right)\left(p^{3 d}+1\right)\end{array}$ \\
\hline$p^{m-1}(p-1)$ & $\begin{array}{l}p\left(1-\mu p^{3 s-d}-\mu p^{3 s-8 d}+p^{m-d}+\right. \\
\left.\underline{p^{2 m}+p^{2 m-9 d}+\mu p^{3 s-3 d}-\mu p^{3 s-5 d}-p^{m-4 d}-p^{m-6 d}}\right)\left(p^{m}-1\right)\end{array}$ \\
\hline$p^{m}$ & $p-1$ \\
\hline
\end{tabular}

- $(i, \lambda)=\left(p^{\frac{m+d-2}{2}}\left(p^{\frac{m-d+2}{2}}-p^{\frac{m-d}{2}}+\varepsilon\right), \frac{1}{2} p^{m-d-1}\left(p^{\frac{m-d+2}{2}}-p^{\frac{m-d}{2}}+\varepsilon\right)[\right.$ $\left.\left.p^{\frac{m+d-2}{2}}\left(p^{\frac{m-d+2}{2}}-p^{\frac{m-d}{2}}+\varepsilon\right)-1\right]\left(p^{\frac{m-d}{2}}+\varepsilon\right)\right)$;

- $(i, \lambda)=\left(p^{\frac{m+2 d-2}{2}}(p-1)\left(p^{\frac{m-2 d}{2}}+\varepsilon\right), \frac{1}{2} p^{s-d-1}\left(p^{\frac{m-2 d}{2}}+\varepsilon\right)\left[p^{\frac{m+2 d-2}{2}}(p-\right.\right.$ 1) $\left.\left.\left(p^{\frac{m-2 d}{2}}+\varepsilon\right)-1\right]\left(p^{m-d}-1\right) /\left(p^{2 d}-1\right)\right)$;

- $(i, \lambda)=\left(p^{\frac{m+2 d-2}{2}}\left(p^{\frac{m-2 d+2}{2}}-p^{\frac{m-2 d}{2}}+\varepsilon\right), \frac{1}{2} p^{s-d-1}\left(p^{\frac{m-2 d+2}{2}}-p^{\frac{m-2 d}{2}}+\right.\right.$ $\left.\varepsilon)\left[p^{\frac{m+2 d-2}{2}}\left(p^{\frac{m-2 d+2}{2}}-p^{\frac{m-2 d}{2}}+\varepsilon\right)-1\right]\left(p^{m-d}-1\right) /\left(p^{2 d}-1\right)\right)$;

- $(i, \lambda)=\left(p^{m-1}(p-1),\left(p^{m}-p^{m-1}-1\right)\left(p^{2 m-d}-p^{2 m-2 d}+p^{2 m-3 d}-\right.\right.$ $\left.\left.p^{m-2 d}+1\right)\right)$.

For $m=6,{\overline{\mathbb{C}^{\perp}}}^{\perp}$ also holds $2-\left(p^{m}, i, \lambda\right)$ designs for the following pairs: 
- $(i, \lambda)=\left(p^{2}(p-1)\left(p^{3}+\varepsilon\right), p^{6}\left(p^{3}+\varepsilon\right)\left[p^{2}(p-1)\left(p^{3}+\varepsilon\right)-1\right]\left(p^{6}\right.\right.$ $\left.\left.-p^{4}-p^{2}+1\right) / 2\left(p^{4}-1\right)\right)$

- $(i, \lambda)=\left(p^{2}\left(p^{4}-p^{3}+\varepsilon\right), \frac{1}{2} p^{6}\left(p^{4}-p^{3}+\varepsilon\right)\left[p^{2}\left(p^{4}-p^{3}+\varepsilon\right)-1\right]\left(p^{6}\right.\right.$ $\left.\left.-p^{4}-p^{2}+1\right) /\left(p^{4}-1\right)\right)$

- $(i, \lambda)=\left(p^{3}(p-1)\left(p^{2}+\varepsilon\right), \frac{1}{2} p^{3}\left(p^{4}-1\right)\left[p^{3}(p-1)\left(p^{2}+\varepsilon\right)-1\right]\right)$;

- $(i, \lambda)=\left(p^{3}\left(p^{3}-p^{2}+\varepsilon\right), \frac{1}{2} p^{3}\left(p^{3}-p^{2}+\varepsilon\right)\left[p^{3}\left(p^{3}-p^{2}+\varepsilon\right)-1\right]\left(p^{2}\right.\right.$ $+\varepsilon))$

- $(i, \lambda)=\left(p^{4}(p-1)^{2}, \frac{1}{2}(p-1)\left[p^{4}(p-1)^{2}-1\right]\right)$;

- $(i, \lambda)=\left(p^{4}\left(p^{2}-p+\varepsilon\right), \frac{1}{2}\left(p^{2}-p+\varepsilon\right)\left[p^{4}\left(p^{2}-p+\varepsilon\right)-1\right]\right)$;

- $(i, \lambda)=\left(p^{5}(p-1),\left(p^{6}-p^{5}-1\right)\left(p^{10}-p^{8}+p^{6}-p^{2}+1\right)\right)$.

(3) If $d^{\prime}=2 d$, then

- $(i, \lambda)=\left(p^{s-1}(p-1)\left(p^{s}-\mu\right), p^{s-1}\left(p^{s}-\mu\right)\left[p^{s-1}(p-1)\left(p^{s}-\mu\right)-\right.\right.$ 1] $\left(p^{m+6 d}-p^{m+4 d}-p^{m+d}+\mu p^{s+5 d}-\mu p^{s+4 d}+p^{6 d}\right) /\left(p^{d}+1\right)\left(p^{2 d}-\right.$ 1) $\left.\left(p^{3 d}+1\right)\right)$

- $(i, \lambda)=\left(p^{s-1}\left(p^{s+1}-p^{s}+\mu\right), p^{s-1}\left(p^{s+1}-p^{s}+\mu\right)\left[p^{s-1}\left(p^{s+1}-p^{s}+\right.\right.\right.$ $\mu)-1]\left(p^{m+6 d}-p^{m+4 d}-p^{m+d}+\mu p^{s+5 d}-\mu p^{s+4 d}+p^{6 d}\right) /\left(p^{d}+1\right)$ $\left.\left(p^{2 d}-1\right)\left(p^{3 d}+1\right)\right)$

- $(i, \lambda)=\left(p^{s+d-1}(p-1)\left(p^{s-d}+\mu\right), p^{s-d-1}\left(p^{s-d}+\mu\right)\left[p^{s+d-1}(p-1)(\right.\right.$ $\left.\left.p^{s-d}+\mu\right)-1\right]\left(p^{m+3 d}+p^{m+2 d}-p^{m}-p^{m-d}-p^{m-2 d}-\mu p^{s+3 d}+\right.$ $\left.\left.\mu p^{s}+p^{3 d}\right) /\left(p^{d}+1\right)^{2}\left(p^{2 d}-1\right)\right)$;

- $(i, \lambda)=\left(p^{s+d-1}\left(p^{s-d+1}-p^{s-d}-\mu\right), p^{s-d-1}\left(p^{s-d+1}-p^{s-d}-\mu\right)[\right.$ $\left.p^{s+d-1}\left(p^{s-d+1}-p^{s-d}-\mu\right)-1\right]\left(p^{m+3 d}+p^{m+2 d}-p^{m}-p^{m-d}-\right.$ $\left.\left.p^{m-2 d}-\mu p^{s+3 d}+\mu p^{s}+p^{3 d}\right) /\left(p^{d}+1\right)^{2}\left(p^{2 d}-1\right)\right)$;

- $(i, \lambda)=\left(p^{s+2 d-1}(p-1)\left(p^{s-2 d}-\mu\right), p^{s-2 d-1}\left(p^{s-2 d}-\mu\right)\left[p^{s+2 d-1}(\right.\right.$ $\left.\left.(p-1) p^{s-2 d}-\mu\right)-1\right]\left(p^{s-d}+\mu\right)\left(p^{s+d}+p^{s}-p^{s-2 d}-\mu p^{d}\right) /\left(p^{d}+\right.$ $\left.1)^{2}\left(p^{2 d}-1\right)\right)$

- $(i, \lambda)=\left(p^{s+2 d-1}\left(p^{s-2 d+1}-p^{s-2 d}+\mu\right), p^{s-2 d-1}\left(p^{s-2 d+1}-p^{s-2 d}+\right.\right.$ $\mu)\left[p^{s+2 d-1}\left(p^{s-2 d+1}-p^{s-2 d}+\mu\right)-1\right]\left(p^{s-d}+\mu\right)\left(p^{s+d}+p^{s}-p^{s-2 d}-\right.$ $\left.\left.\mu p^{d}\right) /\left(p^{d}+1\right)^{2}\left(p^{2 d}-1\right)\right)$

- $(i, \lambda)=\left(p^{s+3 d-1}(p-1)\left(p^{s-3 d}+\mu\right), p^{s-3 d-1}\left(p^{s-3 d}+\mu\right)\left[p^{s+3 d-1}(\right.\right.$ $\left.\left.(p-1) p^{s-3 d}+\mu\right)-1\right]\left(p^{s-2 d}-\mu\right)\left(p^{s-d}+\mu\right) /\left(p^{d}+1\right)\left(p^{2 d}-1\right)\left(p^{3 d}+\right.$ 1));

- $(i, \lambda)=\left(p^{s+3 d-1}\left(p^{s-3 d+1}-p^{s-3 d}-\mu\right), p^{s-3 d-1}\left(p^{s-3 d+1}-p^{s-3 d}-\right.\right.$ $\mu)\left[p^{s+3 d-1}\left(p^{s-3 d+1}-p^{s-3 d}-\mu\right)-1\right]\left(p^{s-2 d}-\mu\right)\left(p^{s-d}+\mu\right) /\left(p^{d}+\right.$ 1) $\left.\left(p^{2 d}-1\right)\left(p^{3 d}+1\right)\right)$

- $(i, \lambda)=\left(p^{m-1}(p-1),\left(p^{m}-p^{m-1}-1\right)\left(1-\mu p^{3 s-d}-\mu p^{3 s-8 d}+p^{m-d}\right.\right.$ $\left.+\frac{p^{2 m}+p^{2 m-9 d}+\mu p^{3 s-3 d}-\mu p^{3 s-5 d}-p^{m-4 d}-p^{m-6 d}}{p^{d}+1}\right)$. 


\section{PROOFS OF THE MAIN RESULTS}

Now, we are ready to present the proofs of our main results. We begin this section by proving the weight distribution of the code $\overline{\mathbb{C}}^{\perp}$.

Proof of Theorem 7 For each nonzero codeword $\mathbf{c}(a, b, c, h)=\left(c_{0}, c_{1}, \ldots, c_{n}\right)$ in $\overline{\mathbb{C}}^{\perp}$, the Hamming weight of $\mathbf{c}(a, b, c, h)$ is

$$
w_{H}(\mathbf{c}(a, b, c, h))=p^{m}-T(a, b, c, h),
$$

where

$$
\begin{gathered}
T(a, b, c, h)=\mid\left\{x \in \mathbb{F}_{q}: \quad \operatorname{Tr}\left(a x^{p^{3 l}+1}+b x^{p^{l}+1}+c x\right)+h=0,\right. \\
\left.a, b, c \in \mathbb{F}_{q}, h \in \mathbb{F}_{p}\right\} \mid .
\end{gathered}
$$

With the orthogonality relations for character sums, and the definitions of $S(a, b, c)$ and $\sigma_{y}$, we have the following equation

$$
\begin{aligned}
& T(a, b, c, h)=\frac{1}{p} \sum_{y \in \mathbb{F}_{p}} \sum_{x \in \mathbb{F}_{q}} \zeta_{p}^{y\left[\operatorname{Tr}\left(a x^{p^{3 l}+1}+b x^{p^{l}+1}+c x\right)+h\right]} \\
& =\frac{1}{p} \sum_{y \in \mathbb{F}_{p}} \zeta_{p}^{y h} \sum_{x \in \mathbb{F}_{q}} \zeta_{p}^{y \operatorname{Tr}\left(a x^{p^{3 l}+1}+b x^{p^{l}+1}+c x\right)} \\
& =p^{m-1}+\frac{1}{p} \sum_{y \in \mathbb{F}_{p}^{*}} \zeta_{p}^{y h} \sigma_{y}(S(a, b, c)) \text {. }
\end{aligned}
$$

If $d^{\prime}=d$ is odd, with the value of $S(a, b, c)$ given in Lemma 5 and the result on cyclotomic fields presented in Lemma 6, we obtain explicitly $\sigma_{y}(S(a, b, c))$. Therefore, plugging $\sigma_{y}(S(a, b, c))$ in the equation above and applying Lemma 4, we have the corresponding value distribution of $T(a, b, c, h)$ given in Table 4 after a tedious calculation, where $j \in \mathbb{F}_{p}$ which is different from that of Lemma 5. Thus, with Table 4 and Eq.(4), we can present the weight distribution of the code $\overline{\mathbb{C}}^{\perp}$ in Table 5 .

With the similar proof idea, we can get Table 6 if $d^{\prime}=d$ is even, and Table 7 if $d^{\prime}=2 d$, respectively.

Thus, we complete the proof of Theorem 7 .

Now, we will use Lemma 1 to prove that $\overline{\mathbb{C}^{\perp}}$ is affine-invariant.

Lemma 10. The extended code $\overline{\mathbb{C}^{\perp}}$ is affine-invariant.

Proof. As one know, $T=C_{1} \cup C_{p^{l}+1} \cup C_{p^{3 l}+1}$ is the defining set of the cyclic code $\mathbb{C}^{\perp}$. Since $0 \notin T$, the defining set $\bar{T}$ of $\overline{\mathbb{C}^{\perp}}$ is given by $\bar{T}=C_{1} \cup C_{p^{l}+1} \cup$ $C_{p^{3 l}+1} \cup\{0\}$. Let $v \in \bar{T}$ and $r \in \mathcal{P}$ such that $r \preceq v$. To applying Lemma 1 , we need to prove $r \in \bar{T}$. 
TABLE 4. The value of $T(a, b, c, h)$ when $d^{\prime}=d$ is odd $\left(j \in \mathbb{F}_{p}\right)$

\begin{tabular}{ll}
\hline Value & Corresponding Condition \\
\hline$p^{m-1}$ & $S(a, b, c)=0$ or $S(a, b, c)=\varepsilon \sqrt{p^{*}} \zeta_{p}^{j} p^{\frac{m-1}{2}}$ and $h+j$ \\
& $=0$ or $S(a, b, c)=\varepsilon \sqrt{p^{*} \zeta_{p}^{j}} p^{\frac{m+2 d-1}{2}}$ and $h+j=0$ \\
$p^{m-1}+\varepsilon(-1)^{\frac{p-1}{2}} p^{\frac{m-1}{2}}$ & $S(a, b, c)= \pm \varepsilon \sqrt{p^{*}} p^{\frac{m-1}{2}} \zeta_{p}^{j}$ and $\eta^{\prime}(h+j) \in\{\varepsilon,-\varepsilon\}$ \\
$p^{m-1}+\varepsilon p^{\frac{m+d-2}{2}}(p-1)$ & $S(a, b, c)=\varepsilon p^{\frac{m+d}{2}} \zeta_{p}^{j}$ and $h+j=0$ \\
$p^{m-1}-\varepsilon p^{\frac{m+d-2}{2}}$ & $S(a, b, c)=\varepsilon p^{\frac{m+d}{2}} \zeta_{p}^{j}$ and $h+j \neq 0$ \\
$p^{m-1}+\varepsilon(-1)^{\frac{p-1}{2}} p^{\frac{m+2 d-1}{2}}$ & $S(a, b, c)= \pm \varepsilon \sqrt{p^{*}} p^{\frac{m+2 d-1}{2}} \zeta_{p}^{j}$ and $\eta^{\prime}(h+j) \in\{\varepsilon,-\varepsilon\}$ \\
$p^{m}$ & $S(a, b, c)=p^{m}$ and $h=0$ \\
0 & $S(a, b, c)=p^{m}$ and $h \neq 0$ \\
\hline
\end{tabular}

TABLE 5. The weight distribution of ${\overline{\mathbb{C}^{\perp}}}^{\perp}$ when $d^{\prime}=d$ is odd

\begin{tabular}{ll}
\hline Value & Multiplicity \\
\hline$p^{m-1}(p-1)$ & $M_{1 v}+\frac{p-1}{2} M_{2 v}+M_{5 v}+\frac{p-1}{2} M_{6 v}$ \\
& $+p M_{7}$ \\
$p^{\frac{m-1}{2}}\left(p^{\frac{m+1}{2}}-p^{\frac{m-1}{2}}+\varepsilon(-1)^{\frac{p-1}{2}}\right)$ & $\frac{p-1}{2} M_{1 v}+\frac{(p-1)^{2}}{2} M_{2 v}$ \\
$p^{\frac{m+d-2}{2}}(p-1)\left(p^{\frac{m-d}{2}}-\varepsilon\right)$ & $M_{3 v}+(p-1) M_{4 v}$ \\
$p^{\frac{m+d-2}{2}}\left(p^{\frac{m-d+2}{2}}-p^{\frac{m-d}{2}}+\varepsilon\right)$ & $(p-1) M_{3 v}+(p-1)^{2} M_{4 v}$ \\
$p^{\frac{m+2 d-1}{2}}\left(p^{\frac{m-2 d+1}{2}}-p^{\frac{m-2 d-1}{2}}+\varepsilon(-1)^{\frac{p-1}{2}}\right)$ & $\frac{p-1}{2} M_{5 v}+\frac{(p-1)^{2}}{2} M_{6 v}$ \\
$p^{m}$ & $p-1$ \\
\hline
\end{tabular}

If $r=0$, it is easily seen that $r \in \bar{T}$. Consider now the case $r>0$. If $v \in C_{1}$, the Hamming weight $w t(v)=1$. It follows from the assumption $r \preceq v$ that $w t(r)=1$, which implies that $r \in C_{1} \subset \bar{T}$. If $v \in C_{p^{l}+1} \cup C_{p^{3 l}+1}$, then the Hamming weight $w t(v)=2$. Since $r \preceq v$, either $w t(r)=1$ or $r=v$. In both cases, $r \in \bar{T}$. The desired conclusion then follows from Lemma 1 .

Proof of Theorem 8 . Theorem 8 is an immediate result of Theorem 2 and Lemma 10.

Proof of Theorem 9 By Theorem 3, it is easy to know that the number of the different supports of all codewords with weight $i \neq 0$ in $\overline{\mathbb{C}}^{\perp}$ is equal to 
TABLE 6. The weight distribution of $\overline{\mathbb{C}}^{\perp}$ when $d^{\prime}=d$ is even

\begin{tabular}{ll}
\hline Value & Multiplicity \\
\hline$p^{s-1}(p-1)\left(p^{s}-\varepsilon\right)$ & $M_{1 v}+(p-1) M_{2 v}$ \\
$p^{s-1}\left(p^{s+1}-p^{s}+\varepsilon\right)$ & $(p-1) M_{1 v}+(p-1)^{2} M_{2 v}$ \\
$p^{\frac{m+d-2}{2}}(p-1)\left(p^{\frac{m-d}{2}}-\varepsilon\right)$ & $M_{3 v}+(p-1) M_{4 v}$ \\
$p^{\frac{m+d-2}{2}}\left(p^{\frac{m-d+2}{2}}-p^{\frac{m-d}{2}}+\varepsilon\right)$ & $(p-1) M_{3 v}+(p-1)^{2} M_{4 v}$ \\
$p^{\frac{m+2 d-2}{2}}(p-1)\left(p^{\frac{m-2 d}{2}}-\varepsilon\right)$ & $M_{5 v}+(p-1) M_{6 v}$ \\
$p^{\frac{m+2 d-2}{2}}\left(p^{\frac{m-2 d+2}{2}}-p^{\frac{m-2 d}{2}}+\varepsilon\right)$ & $(p-1) M_{5 v}+(p-1)^{2} M_{6 v}$ \\
$p^{m-1}(p-1)$ & $p M_{7}$ \\
$p^{m}$ & $p-1$ \\
\hline
\end{tabular}

TABLE 7. The weight distribution of $\overline{\mathbb{C}}^{\perp}$ when $d^{\prime}=2 d$

\begin{tabular}{ll}
\hline Value & Multiplicity \\
\hline$p^{s-1}(p-1)\left(p^{s}-\mu\right)$ & $M_{1}+(p-1) M_{2}$ \\
$p^{s-1}\left(p^{s+1}-p^{s}+\mu\right)$ & $(p-1) M_{1}+(p-1)^{2} M_{2}$ \\
$p^{s+d-1}(p-1)\left(p^{s-d}+\mu\right)$ & $M_{3}+(p-1) M_{4}$ \\
$p^{s+d-1}\left(p^{s-d+1}-p^{s-d}-\mu\right)$ & $(p-1) M_{3}+(p-1)^{2} M_{4}$ \\
$p^{s+2 d-1}(p-1)\left(p^{s-2 d}-\mu\right)$ & $M_{5}+(p-1) M_{6}$ \\
$p^{s+2 d-1}\left(p^{s-2 d+1}-p^{s-2 d}+\mu\right)$ & $(p-1) M_{5}+(p-1)^{2} M_{6}$ \\
$p^{s+3 d-1}(p-1)\left(p^{s-3 d}+\mu\right)$ & $M_{7}+(p-1) M_{8}$ \\
$p^{s+3 d-1}\left(p^{s-3 d+1}-p^{s-3 d}-\mu\right)$ & $(p-1) M_{7}+(p-1)^{2} M_{8}$ \\
$p^{m-1}(p-1)$ & $p M_{9}$ \\
$p^{m}$ & $p-1$ \\
\hline
\end{tabular}

$A_{i} /(p-1)$ for each $i$, where $A_{i}$ is given in Tables 13. Then, from Theorem 8 and Eq.(1), together with a tedious calculation, we can get the desired conclusions.

Example 1. If $(p, m, l)=(3,6,2)$, then the code ${\overline{\mathbb{C}^{\perp}}}^{\perp}$ has parameters $[729,19$, $324]$ and weight enumerator $1+3276 z^{324}+6552 z^{405}+2653560 z^{432}+4245696$ $z^{459}+171950688 z^{468}+343901376 z^{477}+116208456 z^{486}+343901376 z^{495}+$ $171950688 z^{504}+5307120 z^{513}+2122848 z^{540}+6552 z^{567}+3276 z^{648}+2 z^{729}$, which confirms the results given in Theorem 7 
TABLE 8. The value distribution of $S(a, b, c)$ when $d^{\prime}=d$ is odd

\begin{tabular}{ll}
\hline Value & Multiplicity \\
$\varepsilon \varepsilon \sqrt{p^{*}} p^{\frac{m-1}{2}}$ & $M_{1 v}=\frac{p^{m+2 d-1}\left(p^{m}-p^{m-d}-p^{m-2 d}+1\right)\left(p^{m}-1\right)}{2\left(p^{2 d}-1\right)}$ \\
$\varepsilon \zeta_{p}^{j} \sqrt{p^{*}} p^{\frac{m-1}{2}}$ & $M_{2 v}=\frac{p^{2 d}\left(p^{m-1}+\varepsilon \eta^{\prime}(-j) p^{\frac{m-1}{2}}\right)\left(p^{m}-p^{m-d}-p^{m-2 d}+1\right)\left(p^{m}-1\right)}{2\left(p^{2 d}-1\right)}$ \\
$\varepsilon p^{\frac{m+d}{2}}$ & $M_{3 v}=\frac{1}{2} p^{m-d-1}\left(p^{\frac{m-d}{2}}+\varepsilon(p-1)\right)\left(p^{\frac{m-d}{2}}+\varepsilon\right)\left(p^{m}-1\right)$ \\
$\varepsilon \zeta_{p}^{j} p^{\frac{m+d}{2}}$ & $M_{4 v}=\frac{1}{2} p^{m-d-1}\left(p^{\frac{m-d}{2}}-\varepsilon\right)\left(p^{\frac{m-d}{2}}+\varepsilon\right)\left(p^{m}-1\right)$ \\
$\varepsilon \sqrt{p^{*}} p^{\frac{m+2 d-1}{2}}$ & $M_{5 v}=\frac{1}{2} p^{m-2 d-1}\left(p^{m-d}-1\right) /\left(p^{2 d}-1\right)\left(p^{m}-1\right)$ \\
$\varepsilon \zeta_{p}^{j} \sqrt{p^{*}} p^{\frac{m+2 d-1}{2}}$ & $M_{6 v}=\frac{\left(p^{m-2 d-1}+\varepsilon \eta^{\prime}(-j) p^{\frac{m-2 d-1}{2}}\right)\left(p^{m-d}-1\right)\left(p^{m}-1\right)}{2\left(p^{2 d}-1\right)}$ \\
0 & $M_{7}=\left(p^{2 m-d}-p^{2 m-2 d}+p^{2 m-3 d}-p^{m-2 d}+1\right)\left(p^{m}-1\right)$ \\
$p^{m}$ & 1 \\
\hline
\end{tabular}

\section{CONCLUDING REMARKS}

In this paper, via using exponential sums, we first determined the weight distribution of a class of linear codes derived from the cyclic codes related to Dembowski-Ostrom functions. By the properties of affine-invariant codes, we then obtained that $\overline{\mathbb{C}}^{\perp}$ hold 2-designs and determined their parameters explicitly. However, since the case for $m=6$ and $i=p^{4}\left(p^{2}-1\right)$ does not meet the condition of Theorem 3, there remain some unsolved problems concerning the parameters of the 2-designs derived from the supports of all codewords with the weight $i$ in $\overline{\mathbb{C}}^{\perp}$. This may constitute a challenge for future work.

\section{APPENDIX I}

\section{ACKNOWLEDGMENTS}

The authors would like to thank the anonymous referees for their helpful comments and suggestions, which have greatly improved the presentation and quality of this paper. The research of $\mathrm{X}$. Du was supported by NSFC No. 61772022. The research of C. Fan was supported by NSFC No. 11971395.

\section{REFERENCES}

[1] E. F. Assmus Jr. and J. D. Key. Designs and their codes. Cambridge University Press, Cambridge, 1992. 
TABLE 9. The value distribution of $S(a, b, c)$ when $d^{\prime}=d$ is even

\begin{tabular}{ll}
\hline Value & Multiplicity \\
\hline$\varepsilon p^{s}$ & $M_{1 v}=\frac{p^{2 d}\left(p^{m-1}+\varepsilon(p-1) p^{s-1}\right)\left(p^{m}-p^{m-d}-p^{m-2 d}+1\right)\left(p^{m}-1\right)}{2\left(p^{2 d}-1\right)}$ \\
$\varepsilon \zeta_{p}^{j} p^{s}$ & $M_{2 v}=\frac{p^{2 d}\left(p^{m-1}-\varepsilon p^{s-1}\right)\left(p^{m}-p^{m-d}-p^{m-2 d}+1\right)\left(p^{m}-1\right)}{2\left(p^{2 d}-1\right)}$ \\
$\varepsilon p^{\frac{m+d}{2}}$ & $M_{3 v}=\frac{1}{2} p^{m-d-1}\left(p^{\frac{m-d}{2}}+\varepsilon(p-1)\right)\left(p^{\frac{m-d}{2}}+\varepsilon\right)\left(p^{m}-1\right)$ \\
$\varepsilon \zeta_{p}^{j} p^{\frac{m+d}{2}}$ & $M_{4 v}=\frac{1}{2} p^{m-d-1}\left(p^{\frac{m-d}{2}}-\varepsilon\right)\left(p^{\frac{m-d}{2}}+\varepsilon\right)\left(p^{m}-1\right)$ \\
$\varepsilon p^{\frac{m+2 d}{2}}$ & $M_{5 v}=\frac{\left(p^{m-2 d-1}+\varepsilon(p-1) p^{\frac{m-2 d}{2}-1}\right)\left(p^{m-d}-1\right)\left(p^{m}-1\right)}{2\left(p^{2 d}-1\right)}$ \\
$\varepsilon \zeta_{p}^{j} p^{\frac{m+2 d}{2}}$ & $M_{6 v}=\frac{\left(p^{m-2 d-1}-\varepsilon p^{\frac{m-2 d}{2}-1}\right)\left(p^{m-d}-1\right)\left(p^{m}-1\right)}{2\left(p^{2 d}-1\right)}$ \\
0 & $M_{7}=\left(p^{2 m-d}-p^{2 m-2 d}+p^{2 m-3 d}-p^{m-2 d}+1\right)\left(p^{m}-1\right)$ \\
$p^{m}$ & 1 \\
\hline
\end{tabular}

[2] E. F. Assmus Jr. and H. F. Mattson Jr. New 5-designs. J. Combin. Theory, 6:122-152, 1969.

[3] E. F. Assmus Jr. and H. F. Mattson Jr. Coding and combinatorics. SIAM Rev., 16:349388, 1974.

[4] T. Beth, D. Jungnickel and H. Lenz. Design theory. Vol. II, volume 78 of Encyclopedia of Mathematics and its Applications. Cambridge University Press, Cambridge, second edition, 1999.

[5] C. J. Colbourn and R. Mathon. Steiner systems. In Handbook of Combinatorial Designs, Second Edition, pages 128-135. Chapman and Hall/CRC, 2006.

[6] C. Ding. Codes from difference sets. World Scientific, Singapore, 2015.

[7] C. Ding. Designs from linear codes. World Scientific, Singapore, 2018.

[8] C. Ding. Infinite families of 3-designs from a type of five-weight code. Des. Codes Cryptogr., 86(3):703-719, 2018.

[9] C. Ding and C. Li. Infinite families of 2-designs and 3-designs from linear codes. Discrete Math., 340(10):2415-2431, 2017.

[10] C. Ding, C. Tang and D.T. Vladimir. Linear codes of 2-designs associated with subcodes of the ternary generalized Reed-Muller codes. arXiv: 1907.13032 [Math.CO].

[11] K. Ding and C. Ding. A class of two-weight and three-weight codes and their applications in secret sharing. IEEE Trans. Inform. Theory, 61(11):5835-5842, 2015.

[12] X. Du, R. Wang, C. Tang and Q. Wang. Infinite families of 2-designs from two classes of linear codes. arXiv: 1903.07459.

[13] X. Du, R. Wang, C. Tang and Q. Wang. Infinite families of 2-designs from two classes of binary cyclic codes with three nonzeros. arXiv:1903.08153 [math.CO].

[14] X. Du, R. Wang and C. Fan. Infinite families of 2-designs from a class of cyclic codes. J. Comb. Des., 1-14, 2019.

[15] R. W. Fitzgerald and J. L. Yucas. Sums of Gauss sums and weights of irreducible codes. Finite Fields Appl., 11(1):89-110, 2005.

[16] W. C. Huffman and V. Pless. Fundamentals of error-correcting codes. Cambridge University Press, Cambridge, 2003. 
TABLE 10 . The value distribution of $S(a, b, c)$ when $d^{\prime}=2 d$

\begin{tabular}{ll}
\hline Value & Multiplicity \\
\hline$\mu p^{s}$ & $M_{1}=\left(p^{m-1}+\mu(p-1) p^{s-1}\right)\left(p^{m+6 d}-p^{m+4 d}-p^{m+d}+\mu p^{s+5 d}\right.$ \\
& $\left.-\mu p^{s+4 d}+p^{6 d}\right)\left(p^{m}-1\right) /\left(p^{d}+1\right)\left(p^{2 d}-1\right)\left(p^{3 d}+1\right)$ \\
$\mu \zeta_{p}^{j} p^{s}$ & $M_{2}=\left(p^{m-1}-\mu p^{s-1}\right)\left(p^{m+6 d}-p^{m+4 d}-p^{m+d}+\mu p^{s+5 d}\right.$ \\
& $\left.-\mu p^{s+4 d}+p^{6 d}\right)\left(p^{m}-1\right) /\left(p^{d}+1\right)\left(p^{2 d}-1\right)\left(p^{3 d}+1\right)$ \\
$-\mu p^{s+d}$ & $M_{3}=\left(p^{m-2 d-1}-\mu(p-1) p^{s-d-1}\right)\left(p^{m+3 d}+p^{m+2 d}-p^{m}-\right.$ \\
& $\left.p^{m-d}-p^{m-2 d}-\mu p^{s+3 d}+\mu p^{s}+p^{3 d}\right)\left(p^{m}-1\right) /\left(p^{d}+1\right)^{2}$ \\
& $\left(p^{2 d}-1\right)$ \\
$-\mu \zeta_{p}^{j} p^{s+d}$ & $M_{4}=\left(p^{m-2 d-1}+\mu p^{s-d-1}\right)\left(p^{m+3 d}+p^{m+2 d}-p^{m}-p^{m-d}\right.$ \\
& $\left.-p^{m-2 d}-\mu p^{s+3 d}+\mu p^{s}+p^{3 d}\right)\left(p^{m}-1\right) /\left(p^{d}+1\right)^{2}\left(p^{2 d}-1\right)$ \\
$\mu p^{s+2 d}$ & $M_{5}=\left(p^{s-d}+\mu\right)\left(p^{s+d}+p^{s}-p^{s-2 d}-\mu p^{d}\right)\left(p^{m-4 d-1}+\mu\right.$ \\
& $\left.(p-1) p^{m-2 d-1}\right)\left(p^{m}-1\right) /\left(p^{d}+1\right)^{2}\left(p^{2 d}-1\right)$ \\
$\mu \zeta_{p}^{j} p^{s+2 d}$ & $M_{6}=\left(p^{s-d}+\mu\right)\left(p^{s+d}+p^{s}-p^{s-2 d}-\mu p^{d}\right)\left(p^{m-4 d-1}-\mu\right.$ \\
& $\left.p^{m-2 d-1}\right)\left(p^{m}-1\right) /\left(p^{d}+1\right)^{2}\left(p^{2 d}-1\right)$ \\
$-\mu p^{s+3 d}$ & $M_{7}=\left(p^{s-2 d}-\mu\right)\left(p^{s-d}+\mu\right)\left(p^{m-6 d-1}-\mu(p-1) p^{m-3 d-1}\right)$ \\
& $\left(p^{m}-1\right) /\left(p^{d}+1\right)\left(p^{2 d}-1\right)\left(p^{3 d}+1\right)$ \\
$-\mu \zeta_{p}^{j} p^{s+3 d}$ & $M_{8}=\left(p^{s-2 d}-\mu\right)\left(p^{s-d}+\mu\right)\left(p^{m-6 d-1}+\mu p^{m-3 d-1}\right)\left(p^{m}-1\right)$ \\
& $/\left(p^{d}+1\right)\left(p^{2 d}-1\right)\left(p^{3 d}+1\right)$ \\
0 & $M_{9}=\left(1-\mu p^{3 s-d}-\mu p^{3 s-8 d}+p^{m-d}+\right.$ \\
& $\left.\underline{p^{2 m}+p^{2 m-9 d}+\mu p^{3 s-3 d}-\mu p^{3 s-5 d}-p^{m-4 d}-p^{m-6 d}}\right)\left(p^{m}-1\right)$ \\
& 1 \\
$p^{m}$ &
\end{tabular}

[17] K. Ireland and M. Rosen. A classical introduction to modern number theory, volume 84 of Graduate Texts in Mathematics. Springer-Verlag, New York, second edition, 1990.

[18] T. Kasami, S. Lin and W. W. Peterson. Some results on cyclic codes which are invariant under the affine group and their applications. Information and Control, 11:475496, 1967.

[19] G. T. Kennedy and V. Pless. A coding-theoretic approach to extending designs. Discrete Math., 142(1-3):155-168, 1995.

[20] J.-L. Kim and V. Pless. Designs in additive codes over GF(4). Des. Codes Cryptogr., 30(2):187-199, 2003.

[21] J. Luo, S. Ling and C. Xing. Cycle codes and sequences from a class of DembowskiOstrom functions. arXiv: 0902.4509v1 [cs.IT].

[22] R. Lidl and H. Niederreiter. Finite fields, volume 20 of Encyclopedia of Mathematics and its Applications. Cambridge University Press, Cambridge, second edition, 1997. 
[23] F. J. MacWilliams and N. J. A. Sloane. The theory of error-correcting codes. I. NorthHolland Publishing Co., Amsterdam-New York-Oxford, 1977. North-Holland Mathematical Library, Vol. 16.

[24] C. Reid and A. Rosa. Steiner systems $S(2,4, v)$-a survey. The Electronic Journal of Combinatorics, \#DS18:1-34, 2010.

[25] V. D. Tonchev. Codes and designs. In V. Pless and W. C. Huffman, editors, Handbook of coding theory, Vol. I, II, pages 1229-1267. North-Holland, Amsterdam, 1998.

[26] V. D. Tonchev. Codes. In C. J. Colbourn and J. H. Dinitz, editors, Handbook of combinatorial designs, Discrete Mathematics and its Applications (Boca Raton), pages xxii+984. Chapman \& Hall/CRC, Boca Raton, FL, second edition, 2007.

[27] C. Tang, C. Xiang and K. Feng. Linear code with few weights from inhomogeneous quadratic functions. Des. Codes Cryptogr, 83(3):691-714, 2017.

[28] C. Tang, C. Ding and M. Xiong. Steiner systems $S\left(2,4, \frac{3^{m}-1}{2}\right)$ and 2-designs from ternary linear codes of length $\frac{3^{m}-1}{2}$. arXiv:1901.09228 [cs.IT].

[29] M. van der Vlugt. Hasse-Davenport curves, Gauss sums, and weight distributions of irreducible cyclic codes. J. Number Theory, 55(2):145-159, 1995.

[30] M. Xiong. The weight distributions of a class of cyclic codes. Finite Fields Appl., 18:933-945, 2012.

College of Mathematics and Statistics, Northwest Normal UniverSITY, LANZHOU, GANSU 730070, CHINA

E-mail address: rongw113@126.com

College of Mathematics and Statistics, Northwest Normal UniverSITY, LANZHOU, GANSU 730070, CHINA

E-mail address: ymldxn@126.com

School of Mathematics, Southwest Jiaotong University, Chengdu, Sichuan 610000, CHINA

E-mail address: cuilingfan@163.com

School of Computer Engineering and Science, Shanghai University, SHANGHAI, 200444, CHINA

E-mail address: zhniuestaff.shu.edu.cn 